\title{
Dissipative rogue waves: Extreme pulses generated by passively mode-locked lasers
}

\author{
J. M. Soto-Crespo, ${ }^{1}$ Ph. Grelu, ${ }^{2}$ and Nail Akhmediev ${ }^{3}$ \\ ${ }^{1}$ Instituto de Óptica, CSIC, Serrano 121, E-28006 Madrid, Spain \\ ${ }^{2}$ Laboratoire Interdisciplinaire Carnot de Bourgogne, UMR 5209 CNRS, Université de Bourgogne, 9 avenue A. Savary, \\ BP 47870, Dijon Cedex F-21078, France \\ ${ }^{3}$ Optical Sciences Group, Research School of Physics and Engineering, Institute of Advanced Studies, \\ Australian National University, Canberra, Australian Capital Territory 0200, Australia
}

(Received 26 April 2011; published 18 July 2011)

\begin{abstract}
We study numerically rogue waves in dissipative systems, taking as an example a unidirectional fiber laser in a nonstationary regime of operation. The choice of specific set of parameters allows the laser to generate a chaotic sequence of pulses with a random distribution of peak amplitudes. The probability density function for the intensity maxima has an elevated tail at higher intensities. We have found that the probability of producing extreme pulses in this setup is higher than in any other system considered so far.
\end{abstract}

DOI: 10.1103/PhysRevE.84.016604

PACS number(s): 05.45.Yv, 42.65.-k, 47.20.Ky

\section{INTRODUCTION}

The concept of rogue waves describes a unifying idea that applies to a number of extreme phenomena in hydrodynamics, optics, plasma physics, and other fields [1]. It has been studied extensively in the case of ocean waves [2-5], as this is essential for reducing the travel risk for mariners. In recent years, these studies have extended to the areas of optics [6], capillary waves [7], and even finance [8], thus enriching significantly the original concept.

Until now, rogue waves were mostly studied in conservative systems, with the nonlinear Schrödinger equation (NLSE) being the most popular model [2]. However, experience tells us that rogue waves cannot appear without a sufficient energy supply. This seems to be a paradox. Indeed, how a conservative system that conserves energy can generate rogue waves, which require a significant amount of external energy to be pumped into them?

The solution for this paradox is more or less simple. In the case of the NLSE, exact solutions to model rogue waves contained a finite background. Thus, the energy supply for the rogue waves is provided by the infinitely distributed continuous wave which carries an infinite amount of energy. For example, Peregrine solitons [9], which are considered prototypes of rogue waves in the ocean [10] and in optics [11], are always located on a finite background. This background is necessary as it serves as an unlimited energy supply for the rogue waves whenever they appear. Generally speaking, the background is unstable and splits into a multiplicity of pulses. However, the unlimited amount of energy is still there because of the conservative nature of the model.

In the open ocean, the background is created by the winds over the water surface. Indeed, in most cases, rogue waves cannot appear in the quiet ocean. Stormy conditions are one of the essential prerequisites for their generation. Transfer of the background energy into a single strong wave is the main mechanism for generating rogue waves.

Incorporating the energy supply into the nonlinear wave equation instead of using a conservative system seems to be a natural way to further improve the model. Thus, generalizing the model and using a dissipative system with an external energy supply from the very beginning may be useful in considering rogue waves. The first attempts of such generalization have already been done. Montina et al. [12] studied non-Gaussian statistics and extreme waves in a unidirectional optical oscillator pumped with an external light beam. Self-splitting of the beam into small-scale, strongly focused "optical needles" created conditions in which some of these filaments contained significantly more energy than the average, thus generating a specific form of rogue waves.

In the present work, we use a model different from that in [12]. Namely, we consider high-intensity waves in the temporal domain rather than in space. Our system is defined by a set of equations that describes an optical cavity with gain and loss. In this model, we studied the optical field created when gain is high enough to generate a chaotic sequence of a multiplicity of pulses inside the cavity. This sequence of pulses with chaotically distributed amplitudes is in continuous interaction and may form pulses with amplitudes significantly higher than the average. The pulses with the largest amplitude can be considered rogue waves generated this way. We confirmed numerically that their statistics are indeed different from Gaussian. The probability distribution function has a long tail corresponding to high-amplitude waves, which is a direct indication of the presence of rogue waves in the system.

\section{THE MODEL}

In order to be concrete, we consider a typical lumped model of a mode-locked fiber laser [13], shown schematically in Fig. 1. Namely, the ring cavity is composed of three basic components: (1) an erbium-doped fiber (EDF), (2) a single-mode fiber (SMF) with anomalous dispersion, and (3) a saturable absorber, which is followed by an output coupler that splits out a certain amount of the energy accumulated during the round trip.

The evolution of the field envelope, $\psi(z, t)$, in the SMF is governed by the NLSE:

$$
i \psi_{z}+\frac{D}{2} \psi_{t t}+|\psi|^{2} \psi=0
$$




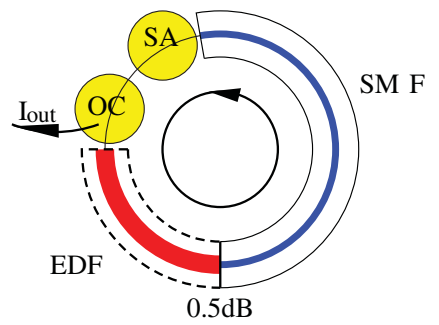

FIG. 1. (Color online) Model of the fiber laser used in our simulations. Notations are as follows: SMF, single mode fiber; SA, saturable absorber; OC, output coupler; and EDF, erbium-doped fiber.

where $D$ is the dispersion parameter, $z$ is the propagation distance, and $t$ is the time in a frame of reference moving with the group velocity, all in normalized dimensionless units.

Pulse propagation in the EDF is modeled by

$i \psi_{z}+\frac{D_{2}}{2} \psi_{t t}+\Gamma_{2}|\psi|^{2} \psi=\frac{i g_{o}}{1+Q / Q_{\text {sat }}}\left(\psi+\beta_{2} \psi_{t t}\right)$,

where the total energy $Q$ is

$$
Q=\int_{-\infty}^{\infty}|\psi|^{2} d t
$$

$Q_{\text {sat }}$ is the parameter representing the saturation energy, $g_{o}$ is the small signal gain, and $\beta_{2}$ represents the spectral width of the gain. $\Gamma_{2}$ is the ratio between the nonlinear coefficients in the EDF and SMF. More precisely, it corresponds to the ratio between the effective mode areas of the EDF and SMF, when both fibers are made of the same glass material, for example, silica.

The instantaneous saturable absorber (SA) is modeled by the following transfer function:

$$
T=T_{o}+\Delta T \frac{I(t)}{I_{\mathrm{sat}}+I(t)},
$$

where $I(t)=|\psi(t)|^{2}, T_{o}$ is the transmission level at low intensities of the optical field, $\Delta T$ is the transmission contrast, and $I_{\text {sat }}$ is the saturation intensity. The output coupler is characterized by the transmission coefficient $T_{r}$. The latter is the coefficient representing power transfer between the two sections of the cavity. A typical $0.5-\mathrm{dB}$ coupling loss is added at the EDF-SMF junction.

The values of the parameters introduced with this model define the mode of operation of the laser system. We can choose the parameters in such a way that the laser operates in one of the following modes: (1) it is a continuous wave (CW) regime, (2) it generates a single stable, stationary pulse every round trip, (3) it generates a periodically oscillating pulse with the period equal to $N$ round trips, (4) it generates several pulses per round trip, or (5) it generates a chaotic field.

Transformation from a CW to pulsing regime in simple distributed models usually occurs when the $\mathrm{CW}$ operation becomes unstable [14]. A similar transition happens in lumped setups. The stationary regime of single-pulse operation is provided by a realistic choice of the model parameters (given in the caption of Fig. 2) and for moderate values of the gain. The latter can be controlled either by $g_{o}$ or by the saturation parameter $Q_{\text {sat }}$. We have chosen $Q_{\text {sat }}$ as the control parameter
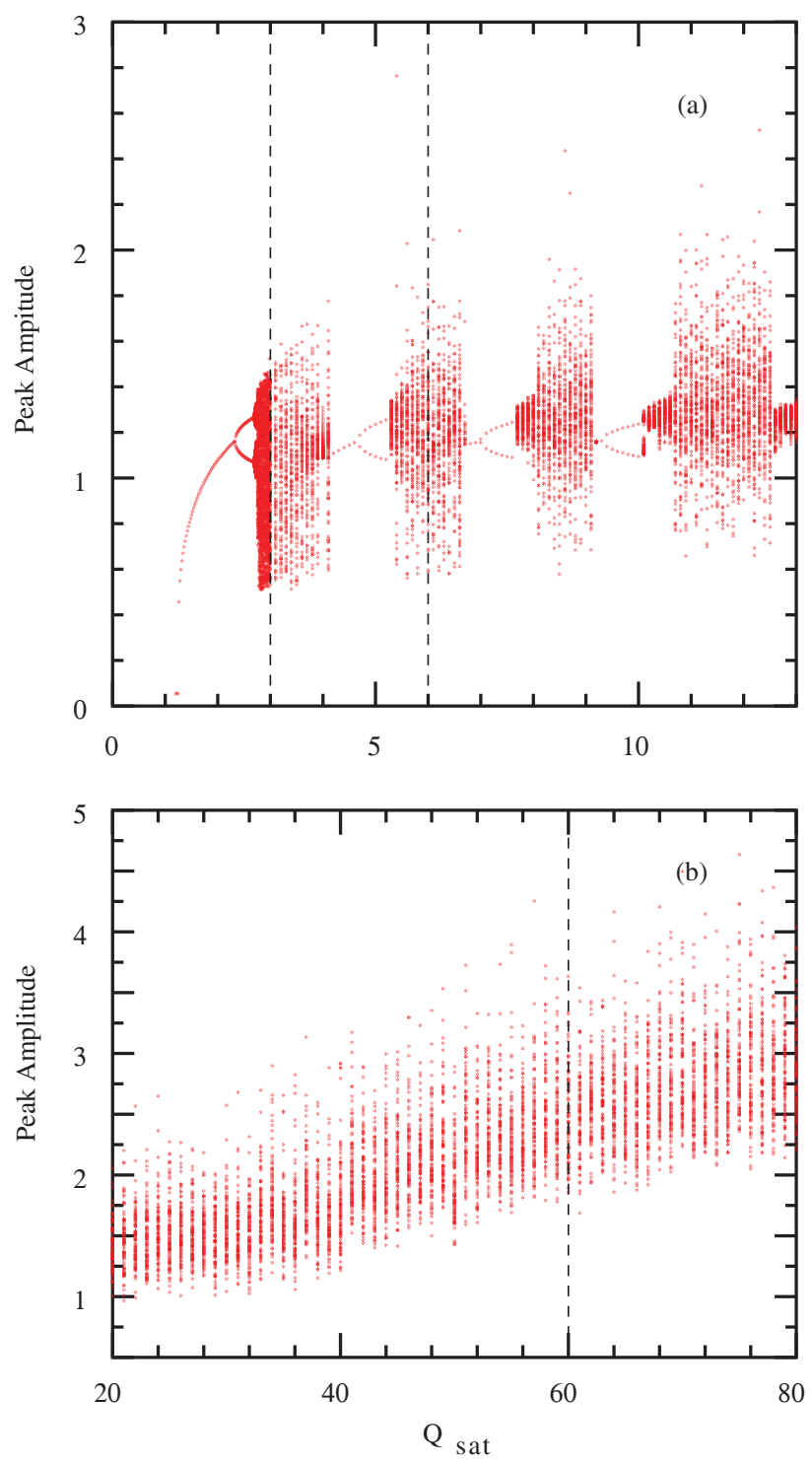

FIG. 2. (Color online) Bifurcation diagram constructed by varying $Q_{\text {sat }}$ while keeping the rest of the parameters fixed. The plot is split into two sections (a) and (b) for convenience. Each (red) dot on the diagram represents the absolute maximum of the field amplitude observed at each round trip. The regions with a single maximum for a given $Q_{\text {sat }}$ in (a) correspond to a stable single-pulse operation. The regions with a wide vertical band represent a chaotic output. These regions appear repeatedly when increasing $Q_{\text {sat }}$. Each of them occupies a finite interval along the $Q_{\text {sat }}$ axis, being a potential area for the appearance of rogue waves. Parameters of the system are $D_{2}=-0.735, \Gamma_{2}=3, g_{0}=4, \beta_{2}=0.008, L_{\mathrm{SMF}}=1.1$, $L_{\mathrm{EDF}}=0.22, T_{r}=70 \%, T_{0}=0.3, \Delta T=0.7$, and $I_{\mathrm{sat}}=0.6$.

as it can be expressed directly in units of the energy pumped into the cavity.

The bifurcation diagram constructed by increasing the value of the control parameter $Q_{\text {sat }}$ is shown in Fig. 2. When decreasing $Q_{\text {sat }}$, we obtained a similar bifurcation diagram with slightly shifted bifurcation points. Certain fixed values of $Q_{\text {sat }}$ provide a chaotic regime independent of the direction of the change. For example, this occurs when the values of $Q_{\text {sat }}$ equal 3,6 , or 60 . We concentrate on these three specific cases, 


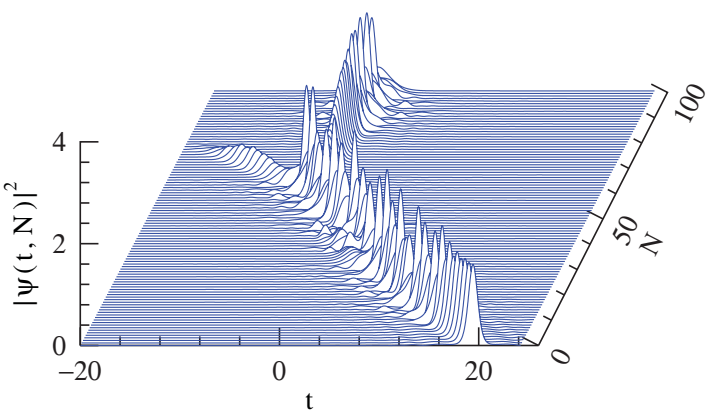

FIG. 3. (Color online) Chaotic evolution of the pulse in the cavity for $Q_{\text {sat }}=3$. The evolution variable, $N$, is the number of round trips.

which are shown in Figs. 2(a) and 2(b) by vertical dashed lines. Analogous bifurcation diagrams are obtained when changing the small signal gain $g_{o}$ rather than $Q_{\text {sat }}$. We do not present them here and concentrate on varying just $Q_{\text {sat }}$.

Sequential transformations between various modes of operation when increasing $Q_{\text {sat }}$ can be seen in the bifurcation diagram of Fig. 2. The laser generates a single pulse with well-defined amplitude at $Q_{\text {sat }}$ between 1.25 and 2.34. The pulse switches amplitude between two fixed values after every round trip when $Q_{\text {sat }}$ increases above 2.34. This transition corresponds to the period doubling of the single-pulse regime [15]. There are several such transitions in Fig. 2(a) with increasing number of pulses inside each round trip. Namely, the laser generates two pulses when $Q_{\text {sat }}$ is around 4.5. It generates three pulses when $Q_{\text {sat }}$ is around 7, and so on. The switch to a multipulse regime occurs when the laser operates in the anomalous path-averaged dispersion, which is the case in all simulations performed here.

At each set of transitions, period doubling is followed by period quadrupling, and after a sequence of transformations the dynamics switches to a chaotic regime when the pulses randomly change amplitude after each round trip. Welldeveloped chaotic behavior of a single pulse can be observed at $Q_{\text {sat }}=3$. An example of this dynamics is shown in Fig. 3. For this value of $Q_{\text {sat }}$, the model does not allow an increase of the number of pulses. Splitting of a single pulse does not multiply the number of pulses as any additional pulse in the cavity dissipates and only one of them survives. At the next region of chaotic dynamics when $Q_{\text {sat }}$ is around 6, we can observe the random evolution of mainly two pulses in each round trip. For the chosen set of parameters, the number of pulses in the cavity is roughly proportional to $Q_{\text {sat }}$ divided by 3 .

For higher values of $Q_{\text {sat }}$, the number of pulses in the cavity increases. The interaction between them produces significantly more complex field profiles. Depending on the position of $Q_{\text {sat }}$ within each interval of chaotic behavior, the pulses may repel or attract each other. For example, when $Q_{\text {sat }}$ is around 30, the pulses mainly repel each other. They tend to be distributed more or less uniformly within the cavity. The dynamics reveals their chaotic motion and collisions. The dynamics within the cavity at $Q_{\text {sat }}=30$ does not reveal any special features. Thus, it is not presented here.

For other values, the pulses have the tendency to group into bunches. An example of this behavior is shown in Fig. 4

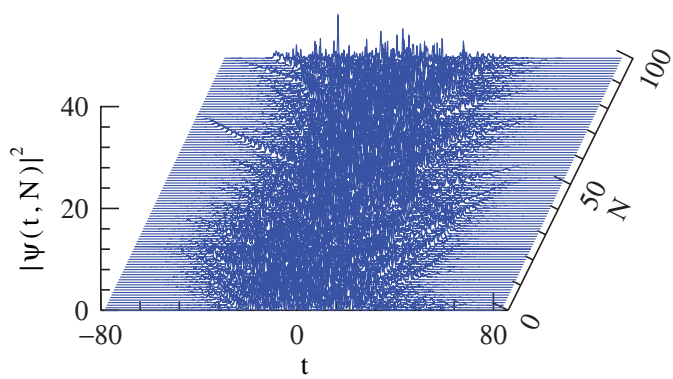

FIG. 4. (Color online) Chaotic evolution of a group of pulses when $Q_{\text {sat }}=60$. Note the different scale in the $t$ axis with respect to Fig. 3 .

obtained for $Q_{\text {sat }}=60$. Here we can observe simultaneously around 20 main pulses with chaotically changing amplitudes. The bunch stays isolated, and pulses that separate from the group eventually vanish. These pulses are submerged into a sea of small-amplitude radiation waves. Then the whole output radiation generated by the laser can be considered completely chaotic. We stress that the specific form of the initial conditions does not influence directly the long-term evolution of the group. We observe similar results starting either from a single Gaussian beam of arbitrary width or a set of individual well-separated bell-shaped pulses. Initial conditions are forgotten after a few round trips, and then the group dominates in the subsequent dynamics.

This regime is ideal for studying rogue waves. Pulse amplitudes vary in large intervals from very small amplitudes to completely unexpected high rises. One example is shown in Figs. 5 and 6. For each $N$, we traced the pulse with the highest amplitude in $t$ domain. In Fig. 5, this peak amplitude is plotted as a function of $N$. Using such diagrams, we detected the tallest pulse in any particular run that starts with chaotic initial conditions.

A section of the optical field profile containing the tallest pulse for this special value of round trip, $N=276$, is shown in Fig. 6. We can see that the pulse intentionally positioned in the middle of the interval, $t=0$, can be considered as a rogue wave as its maximum intensity is almost three times higher than the maximum intensity of the pulse that is next in height. This example shows clearly the random appearance of rogue waves in our model. Higher peak amplitudes can also be observed, but the probability of their appearance is smaller.

\section{STATISTICS OF THE PULSES}

The statistical properties of radiation can be studied either using the whole bunch of pulses or just isolating a finite interval within the bunch. In either case, we obtained identical results. Another example of a pulse sequence obtained for the case $Q=60$ is shown in Fig. 7. It is obtained for the same set of parameters as Fig. 6, but due to the randomness of the process, the average pulse intensities here are lower. In this sense, it can be considered as a more typical output profile among the whole set presented in Fig. 4.

A part of the same realization contained in the red box is presented in Fig. 8. Fivefold magnification in the time scale allows us to see more clearly the shapes of separate pulses. Typically, we used 65536 points to describe the total temporal 

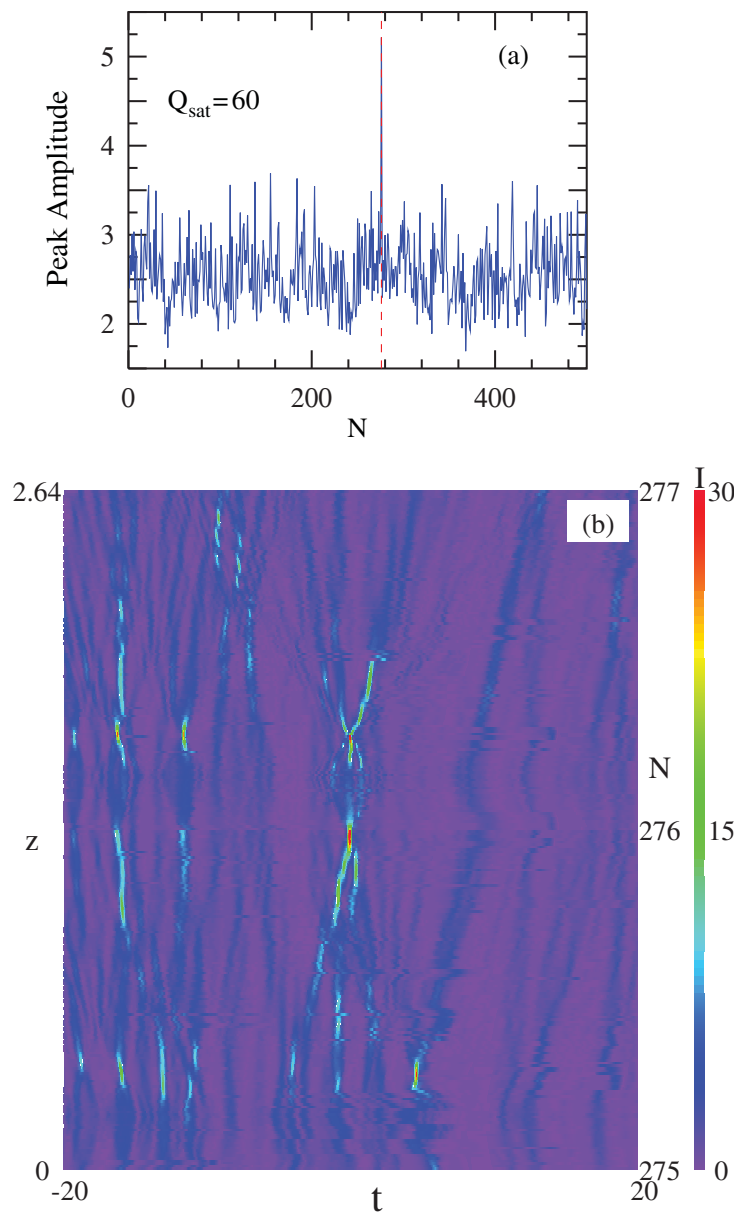

FIG. 5. (Color online) (a) Evolution of the absolute maximum amplitude of the field in $t$ with the number of round trips, $N$, for $Q_{\text {sat }}=60$ (solid blue line). The highest maximum in this run is observed when $N=276$. This value is marked by the dashed (red) vertical line. (b) The contour plot of the same simulation in the $(t, z)$ plane, that is, inside the cavity for the two nearest round trips. Each of the two focused spots (red online) here (at $t=0$ ) is a signature of a rogue wave. The output radiation contains only those formed at the coupler position, that is, at the integer values of $N$.

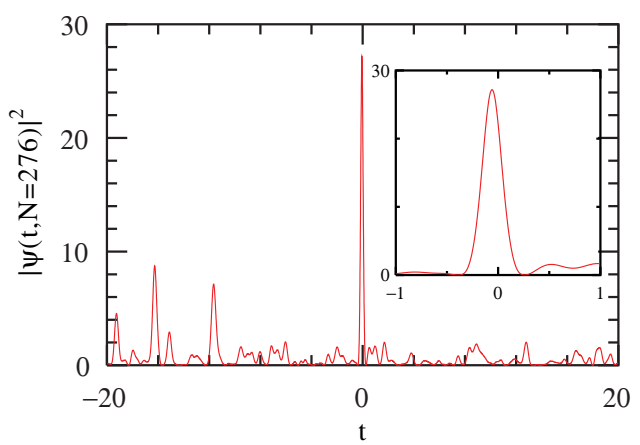

FIG. 6. (Color online) Main segment of the transverse field profile of the laser radiation at the round-trip number $N=276$. The whole bunch of pulses occupies the larger temporal interval $[-50,50]$. The extreme pulse in the middle can be considered as a rogue wave. The inset shows the same pulse in higher resolution.

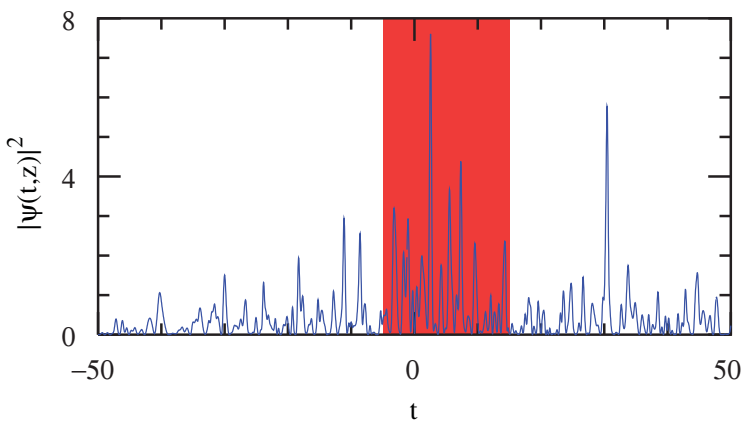

FIG. 7. (Color online) A sample of the output radiation from the model laser. Parameters are the same as in the caption of Fig. 2 with $Q_{\text {sat }}=60$.

interval of $[-400,400]$. This means that every individual pulse is smoothly approximated within the numerical grid which is a necessary condition for having accurate simulations. The solid red curve in this figure represents the envelope of the output field intensity, while the dashed green vertical lines show the position of the field maxima. We counted these maxima in order to calculate the probability density function (PDF) of the output field.

PDFs can be constructed in various ways. For ocean waves, it is customary to count the wave height as the height from trough to crest. This measure has its justification in the action the wave produces on ships. In optics, the natural measure of the pulse height is its maximal intensity relative to the zero level. This is a quantity usually determined by the optical measuring equipment. Thus, it would be natural to construct the PDF calculating the intensity heights of the maxima in the sequences; a typical example is the one shown in Fig. 8.

On the other hand, the notion of rogue wave derived from the oceanic terminology and, in principle, we can also easily calculate the pulse height as the height from trough to crest of intensity, thus allowing for certain comparisons between optical rogue waves and the oceanic ones. This exercise would make sense if we are using the optical rogue waves as a smallscale model for oceanic ones. Additionally, the comparison of various techniques in constructing the PDF is of significant value by itself.

Such comparison is based on the fact that nonlinear waves in optics as well as deep-water waves in the ocean are described by the same nonlinear Schrödinger equation. This common description may call for direct analogies between them.

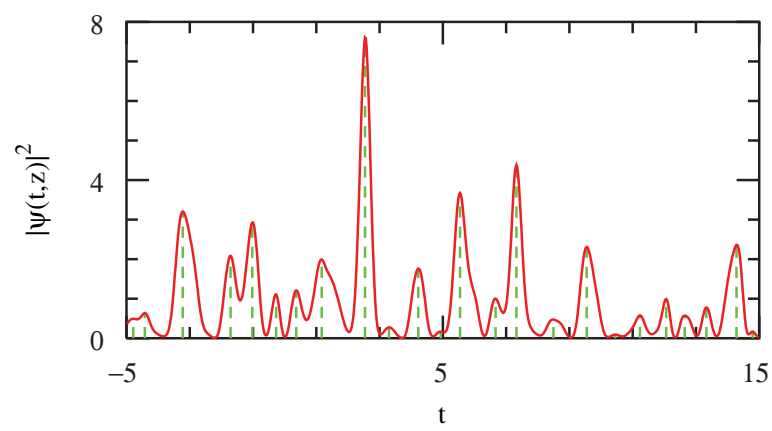

FIG. 8. (Color online) Part of the curve enclosed in the central (red) box in Fig. 7. 
However, even if the equation is the same, the measurable quantities are different. Namely, in optics, we measure the intensity, that is, the square modulus of the amplitude of the envelope of the carrier wave, which is a complex field. In the case of deep-water waves, the carrier wave is a real function. A measurable quantity is the real part of the envelope multiplied by the carrier wave. This difference leads to a significant distinction between the two types of waves. Direct comparisons should be done with care.

Although for conventional laser applications, people have been mostly interested in low-noise CW radiation on the one hand and stable single-pulse mode locking on the other hand, there is presently a growing interest in studying chaotic laser radiation. A decade after the intensive studies of the statistical properties of transverse laser modes [16], research has focused on the statistical properties of single transverse-mode laser emitters. Indeed, chaotic quasi-CW oscillators could be applied in optical cryptography for secure communications [17], whereas noiselike, wide-band laser pulses can be useful for low-coherence reflectometry [18]. However, the generation of a truly chaotic field in the temporal domain is still largely mysterious. Although pulse generation in mode-locked lasers is presently well understood, the recent focus on excitable dynamics within the multiple-pulse quasi-mode-locked regime stimulates further interest in exploration of this type of systems [19].

Generally, the statistics of laser radiation is measured as the second factorial moment [20]. Roughly speaking, the main value of interest is the intensity distribution of the output radiation. Depending on the model, this distribution can look Gaussian [20] or can be a more complicated function of the intensity [21]. When studying rogue waves, we are dealing with the distribution of the maxima of radiation rather than radiation at every point. We expect that the density of maxima should be different from the density of probability at every point of radiation.

\section{PROBABILITY DENSITY FUNCTION}

The bifurcation diagram in Fig. 2 reveals many regions of chaotic dynamics in our laser system. They all have different statistical characteristics. The dynamics depends to a great extent on the number of pulses that exist simultaneously in the cavity. In order to show this, in Fig. 9, we compare the output laser pulses obtained for two values of the parameter $Q_{\text {sat }}$, namely, 3 and 6. In one case $\left(Q_{\text {sat }}=3\right)$, the output signal consists mainly of a single pulse of random amplitude, while in the other case ( $\left.Q_{\text {sat }}=6\right)$, it consists of two main pulses per round trip, each one with random amplitude.

In order to study the statistical properties of the output radiation in each of these cases, we accumulated data over a large number of round trips. The temporal signal has been taken after the output coupler, which corresponds to a stroboscopical measurement process as it would be implemented in the frame of a realistic experiment. We constructed the probability density functions for all local peak intensities $I_{\text {peak }}$ exceeding the threshold of 0.2 . The two probability density functions are shown in Fig. 10.

There is a clear difference between the curves. Namely, the single-pulse dynamics does not allow for high field intensities.
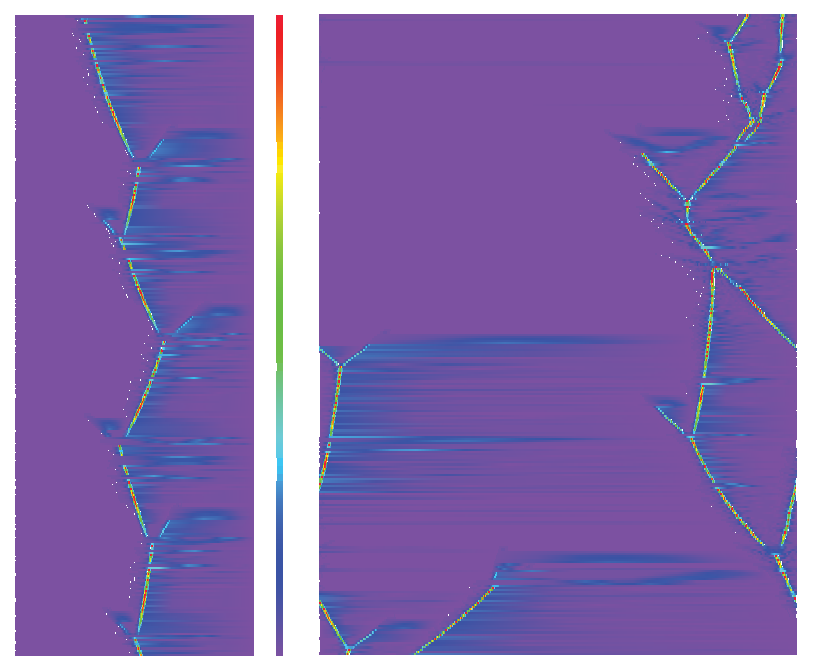

FIG. 9. (Color online) Contour plots of evolution of two sample pulses when $Q_{\text {sat }}=3$ (left) and $Q_{\text {sat }}=6$ (right). The vertical axis corresponds to $N$, and the horizontal axis corresponds to $t$.

Pulses with intensity above 2.8 virtually do not exist. On the other hand, the appearance of a second pulse in the cavity stimulates the growth of the intensity of each of them. Then the corresponding PDF has significant values at intensities higher than 2.8 (blue curve).

This growth of the probability for intensities above 2.8 is related first to the asymmetric distribution of the energy between the two pulses and second to the pulse interaction. The two pulses move chaotically across the cavity and unavoidably collide. Collisions are inelastic in nonintegrable systems, thus allowing for energy exchange between the pulses. Moreover, the pulses can merge or multiply. Such interactions may create the pulses that have higher intensities in comparison with the case of the single-pulse evolution. The shape of the PDF for $Q_{\text {sat }}=6$ in Fig. 10 reveals the appearance of such high-intensity pulses. In fact, the highest intensities are increased more than twice in comparison with the case $Q_{\text {sat }}=3$.

The appearance of pulses with high intensity suggests that these can be identified as rogue waves in dissipative systems.

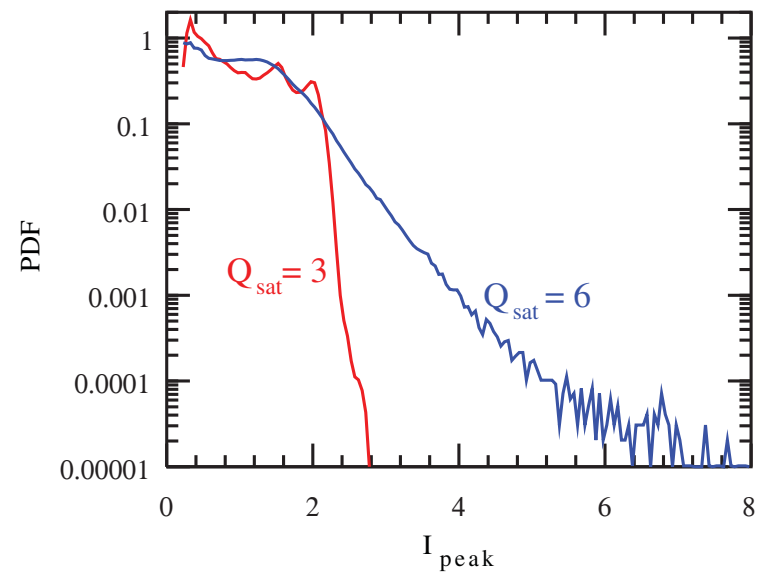

FIG. 10. (Color online) Probability density functions for $Q_{\text {sat }}=3$ (red curve) and $Q_{\text {sat }}=6$ (blue curve). 


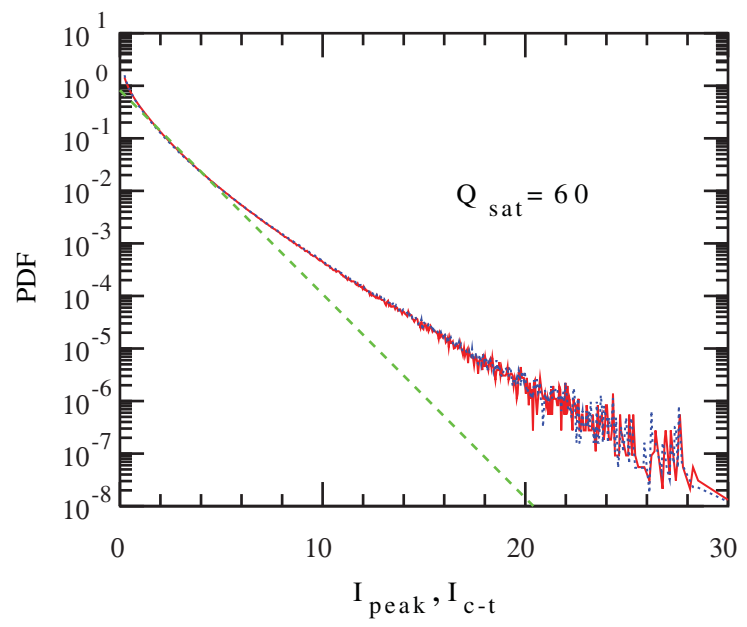

FIG. 11. (Color online) Probability density function for the maxima of intensity (red curve) and for the pulse hight from crest to trough (blue line). The two curves are virtually identical. For comparison, the dashed (green) line is drawn for a pure exponential decay of the probability.

We can call them dissipative rogue waves. As the number of pulses in the cavity increases, the chances for their interaction also become significantly higher. This means that as the value of $Q_{\text {sat }}$ becomes larger, the probability of finding a rogue wave with large intensity becomes higher.

For the value $Q_{\text {sat }}=60$, the probability density function is shown in Fig. 11. It is calculated in two different ways. The dotted (blue) curve in Fig. 11 shows the PDF which has been constructed using as the definition of the wave height the intensity from crest to trough in Fig. 8. The solid (red) curve on the same figure shows the PDF constructed using the maxima of the signal intensity counted from the zero level in Fig. 8. As we can see, qualitatively and quantitatively, the two curves are nearly identical. High-intensity parts of the two curves (above 20) have larger variations as the total amount of data here is much less than for the lower intensities. Unlike for the case of water waves, the PDF can be calculated based on the pulse intensity relative to the zero level. This is a measurable quantity and its use is more natural in optics.

Our results demonstrate that the high-intensity parts of the PDF are indeed elevated. The two curves in Fig. 11 are not straight lines as is expected for any of the classic distributions. For the sake of comparison, in Fig. 11, we also drawn a straight dashed (green) line fitted to approximate the low- to mediumintensity part of the curves. The data at the high-intensity part of the curves are orders of magnitude above this line. Thus, high-intensity events occur more often than they should according to the Gaussian or Rayleigh distributions.

A tricky feature of our plots in Fig. 11 is that the probability of small-amplitude pulses tends to infinity. In optical experiments [6], the low-amplitude pulses are usually eliminated completely from the measurements in order to avoid such difficulties. Then the presence of rogue waves manifests itself in L-shaped curves for the PDFs. However, to be accurate, we have to account for all data rather than just for the high-amplitude waves.

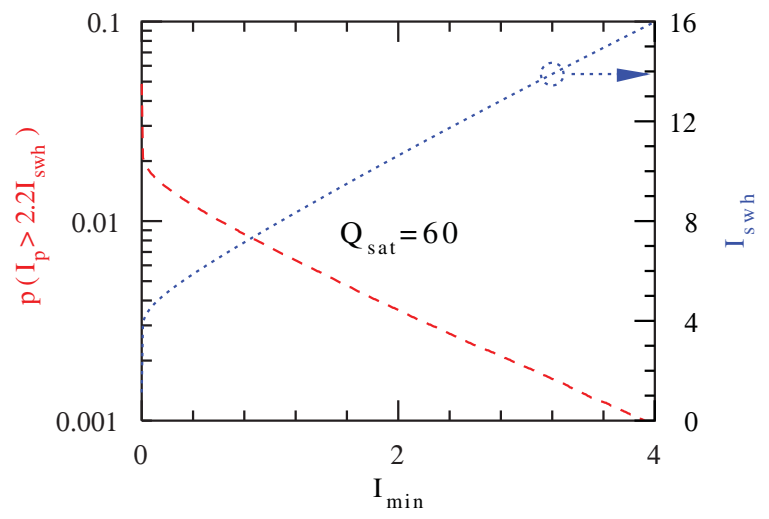

FIG. 12. (Color online) Significant wave height shown by the dotted (blue) curve versus the low-intensity threshold, $I_{\min }$, when the maxima below $I_{\min }$ are not accounted for. The probability of having a pulse with a peak intensity above the level of 2.2 times the significant wave height, $I_{\mathrm{SWH}}$ is shown by the dashed (red) line.

An important issue in defining rogue waves is the level of the significant wave height (SWH). The latter requires the knowledge of the probability of appearance of waves of all amplitudes, starting from the zero level. This is problematic as the PDF in our case does not have a constant limit at low intensities. Instead, it increases to high value that may depend on many factors, including the size of the numerical grid. Thus, counting the total number of events required for the calculation of the SWH is complicated. In order to overcome this difficulty, we have to remove small-intensity pulses below a certain threshold from consideration. This threshold, say $I_{\min }$, is one of the essential parameters in the definition of the SWH and consequently of the rogue wave.

In order to show that, we calculated the SWH for various levels of the threshold intensity, $I_{\min }$. The results are shown in Fig. 12. Here, the blue dotted line represents the SWH versus $I_{\min }$. Despite the fact that the SWH decreases indefinitely when we decrease the $I_{\min }$, the straight-line approximation from the high-intensity part of the curve provides us with the rough estimate of the SWH being around 4. This approximate value can be used for the definition of the minimum intensity of a rogue wave, which is normally (using the common definition taken for ocean waves) 2.2 times the SWH, that is, 8.8 in our case.

The red dashed line in Fig. 12 shows the probability of the appearance of a pulse with an intensity above this level. As we can see from the figure, this probability is relatively high. Even if we choose an unreasonably high value of $I_{\min }$, ignoring most of the low-amplitude pulses, this probability is above 0.001 . The lower limit of the probability is above 0.01 . Thus, extreme pulses in dissipative systems may appear quite often. Roughly speaking, every pulse out of a hundred can be counted as being extreme. This probability is significantly higher than in any of the systems considered so far.

In conclusion, we considered a typical fiber laser cavity in a regime of generating multipulse sequences. At certain sets of parameters, the laser can serve as an example of a dissipative system which transforms a continuous pump of energy into a chaotic train of pulses with random amplitudes. We have 
found that the probability of producing extreme pulses in this laser is higher than in other systems.

\section{ACKNOWLEDGMENTS}

J.M.S.C. acknowledges support from the Spanish Ministerio de Ciencia e Innovación under contract FIS2009-
09895. Ph.G. acknowledges support from the Agence Nationale de la Recherche in the frame of the project ANR2010-BLANC-0417-01-SOLICRISTAL. N.A. acknowledges the support of the Australian Research Council (Discovery Project DP110102068). N.A. acknowledges support from the Humboldt Foundation.
[1] N. Akhmediev and E. Pelinovsky, eds., special issue of Eur. Phys. J. 185 (2010).

[2] K. B. Dysthe and K. Trulsen, Phys. Scr., T 82, 48 (1999).

[3] I. Ten and H. Tomita, Reports of RIAM Symposium No. 17SP1-2 (Nature Publishing Group, London, 2006).

[4] V. V. Voronovich, V. I. Shrira, and G. Thomas, J. Fluid Mech. 604, 263 (2008).

[5] D. Clamond, M. Francius, J. Grue, and C. Kharif, J. Mech. B 25, 536 (2006).

[6] D. R. Solli, C. Ropers, P. Koonath, and B. Jalali, Nature (London) 450, 1054 (2007).

[7] M. Shats, H. Punzmann, and H. Xia, Phys. Rev. Lett. 104, 104503 (2010).

[8] Z.-Y. Yan, Commun. Theor. Phys. 54, 947 (2010).

[9] D. H. Peregrine, J. Aust. Math. Soc. Series B 25, 16 (1983).

[10] V. I. Shrira and V. V. Geogjaev, J. Eng. Math. 67, 11 (2010).

[11] B. Kibler, J. Fatome, C. Finot, G. Millot, F. Dias, G. Genty, N. Akhmediev, and J. M. Dudley, Nat. Phys. 6, 790 (2010).
[12] A. Montina, U. Bortolozzo, S. Residori, and F. T. Arecchi, Phys. Rev. Lett. 103, 173901 (2009).

[13] M. J. Lederer, B. Luther-Davies, H. H. Tan, C. Jagadish, N. Akhmediev, and J. M. Soto-Crespo, J. Opt. Soc. Am. B 16, 895 (1999).

[14] J. M. Soto-Crespo, N. Akhmediev, and G. Town, J. Opt. Soc. Am. B 19, 234 (2002).

[15] J. M. Soto-Crespo, M. Grapinet, Ph. Grelu, and N. Akhmediev, Phys. Rev. E 70, 066612 (2004).

[16] F. T. Arecchi, Physica D 51, 450 (1991).

[17] G. D. Van Wiggeren and R. Roy, Science 279, 1198 (1998).

[18] M. Horowitz, Y. Barad, and Y. Silberberg, Opt. Lett. 22, 799 (1997).

[19] S. Chouli and Ph. Grelu, Phys. Rev. A 81, 063829 (2010).

[20] F. Arecchi, G. S. Rodari, and A. Sona, Phys. Lett. A 25, 59 (1967)

[21] H. Hodara, Proc. IEEE 53, 696 (1965). 